\title{
Mining for Patterns of Semantic Link Usage: Do Domain Users Actually Like Semantic Browsing?
}

\author{
Ed de Quincey, Helen Oliver, Patty Kostkova, \\ Gawesh Jawaheer, Gemma Madle, Gayo Diallo \\ City eHealth Research Centre (CeRC), City University \\ London, UK \\ ed.de.quincey@city.ac.uk, helen.oliver.1@ city.ac.uk, \\ patty@soi.city.ac.uk, gawesh.jawaheer.1@city.ac.uk, \\ g.c.madle@city.ac.uk, diallog@ensma.fr
}

\author{
Bianca Habermann \\ Scionics Computer Innovation \\ Dresden, Germany \\ habermann@mpi-cbg.de
}

\author{
Simon Jupp, Robert Stevens \\ University of Manchester, UK \\ simon.jupp@manchester.ac.uk, \\ robert.stevens@manchester.ac.uk
}

\author{
Dimitra Alexopoulou, Michael Schroeder \\ Biotechnologisches Zentrum \\ TU Dresden, Germany \\ dimitra.alexopoulou@biotec.tu-dresden.de, \\ ms@biotec.tu-dresden.de
}

\begin{abstract}
A perceived limitation of the current Web is that it is comprised of static links with no connection to any underlying domain knowledge. The Semantic Web is seen as a potential solution to this problem by delivering semantically related information to users dynamically. However, the benefit to users is rarely questioned and there have been few real- world user evaluations of semantic systems. In this paper we present a usercentred evaluation of three Semantic Web Browsers (SWB) that have been extended as part of the Sealife project. The results presented are based on analysis of the server logs from each application in relation to time taken to perform pre-defined tasks along with the amount of semantic activity carried out. It was found that the user experience was dependent on the SWB used but there was some indication users will be able to find information more quickly and that users will explore semantic features if present.
\end{abstract}

\section{Semantic Web; Web Log Analysis; User-centred Evaluation}

\section{INTRODUCTION}

One of the characteristics of the current Web is the static nature of hypertext, in which the links have no connection to any underlying domain knowledge [1,2,3]. A popular solution for this problem in the Semantic web community is to model underlying domain knowledge in the form of ontologies or taxonomies, which map objective relationships within the subject matter or domain discussed in the content of a Web site. As the number of available ontologies increases, as is happening in the life sciences [4], it is expected that SW technologies will be adopted as opportunities to use them increase. The assumption that users, presented with SW technologies, will readily perceive the benefits is not often questioned: relatively little attention has been paid to evaluating the perceptions of SW technologies by real-world users. In this paper we present the results of a user-centric evaluation of provision of Semantic links on three district Semantic Web browsers (SWBs) from the Sealife project [5], which has utilised web log analysis techniques to evaluate user behaviour.

\section{The Sealife Semantic Web BRowsers}

Three SWBs have been evaluated in the Sealife project: COHSE, CORESE and GoPubMed.

The COHSE [3,6] service uses an ontology (in this case the NeLI vocabulary [1]) to highlight terms from the ontology that appear on a web page. When a user clicks on a highlighted term, a link box appears, containing links to trusted information sources on the World Wide Web. The links are grouped according to their relationships within the ontology (e.g. has_symptom/is_symptom_of, or broader/narrower).

The CORESE-based SWB [7,8] uses a graphical representation of the underlying ontology to help users to navigate through the NeLI DL. When a user clicks on a node or edge in the graph, relevant documents are presented in a tabbed pane, arranged according to the relevant terms' position in the ontology's hierarchy.

GoPubMed [9] is a semantic search technology applied to the PubMed search engine [10]. GoPubMed makes use of name disambiguation to tell authors apart, and presents search results in the categories What, Who, Where, and When, categorizing them in terms of subject matter, authors, geograp 


\section{AIMS AND OBJECTIVES}

A number of hypotheses were formulated during the development of the SWBs in relation to the improvements offered, compared to non-semantic control platforms:

- The SWB reduces the time taken for users to find information or perform tasks.

- Where semantic links are available, users will follow them instead of non-semantic links.

It was these hypotheses that were then tested during the evaluation described in the following section.

\section{METHODS}

\section{A. Respondents}

COHSE was evaluated by 67 participants, of whom 25 were healthcare professionals, 10 were information professionals, and 32 were from other fields including 14 students. The CORESE-based SWB was evaluated by 28 participants, of whom 11 were healthcare or biological science practitioners, 3 were information professionals, and 4 were from other fields. GoPubMed was evaluated by 141 participants, 21 of whom were biologists.

\section{B. Evaluation Format}

Each SWB was evaluated individually, and compared against its respective control platform, the NeLI Digital Library for COHSE and CORESE, and PubMed for GoPubMed.

The evaluations, all in web format, took place both online and in workshop settings. Each evaluation began with a prequestionnaire regarding the user's occupation and previous experience with the respective control platform. There followed a series of information-seeking tasks appropriate to the nature of each respective browser, followed by two questions, about information findability and ease of use. The evaluation closed with a post-questionnaire comparing the usability of the control platform and the intervention SWB. Respondents either completed a short format of two tasks using the SWB and two tasks using the control system or a long format, which consisted of five tasks with each system. The results form the short format evaluations are presented in this paper.

\section{Weblog Analysis}

During the evaluation, each respondent was assigned a unique identifier (uID) which was passed between each page of the evaluation and the SWB and the NeLI or GoPubMed website via the url quersystring.

The respondents' actions were analysed by extracting relevant log entries that contained the unique identifier from a combination of the logs produced by the SWBs and the server at City University (which hosted the questionnaire and the NeLI website). These entries were processed using a number of bespoke PHP scripts that cleaned the data by removing unnecessary logged activities and then inserting the data into a MYSQL database. SQL queries were then written to extract various information such as times taken for each task, numbers of links clicked etc.. These queries were then outputted as CSV files for further analysis.
During this process it was found that a number of respondents, had started the evaluation and not completed it or had completed it but in an unrealistic time. These users were filtered out and are not presented in the following results section.

\section{RESULTS}

\section{A. COHSE}

1) Time taken for users to find information/perform tasks: The time taken for each task was calculated from the online evaluation logs. The following table describes the average time and range of times taken in minutes for each task for the twenty-four respondents.

TABLE I. AVERAGE TIMES AND RANGES FOR EACH TASK IN SECONDS

\begin{tabular}{|c|l|l|l|l|}
\hline \multirow{2}{*}{} & \multicolumn{2}{|c|}{ COHSE } & \multicolumn{2}{c|}{ NeLI } \\
\cline { 2 - 5 } & \multicolumn{1}{|c|}{ Task 1 } & \multicolumn{1}{c|}{ Task 2 } & Task 1 & \multicolumn{1}{c|}{ Task 2 } \\
\hline Average & 648 & 399 & 544 & 322 \\
\hline Maximum & 4774 & 1766 & 5898 & 2094 \\
\hline Minimum & 55 & 38 & 49 & 59 \\
\hline
\end{tabular}

Sixteen users spent longer on the COHSE tasks than on the NeLI tasks and eight users spent longer on the NeLI tasks than on the COHSE tasks.

2) Use of semantic links compared with non-semantic links: An indication of the use of the semantic links that COHSE provides is the number of times a highlighted term is clicked and the links box is activated and then a further indication is how many views of external sites via COHSE there were. In total one hundred and thirty two sites external to NeLI were viewed via COHSE from ninety-seven link box activations.

The number of link box activations ranged from fourteen to one and there were six users who did not click on any highlighted terms and therefore did not use any of the semantic features.

The number of external pages that were viewed by each user ranged from forty-two to one with eleven users not viewing any external pages.

3) Summary: It is apparent that users spent more time on tasks when using COHSE than on the NeLI alone tasks. This was not the case for all users however and for the second task using COHSE, the average time spent on the task was in fact less overall. This could indicate that for some users, once they had become accustomed to using it, they were more able to take advantage of some of the added features it provides.

Although a number of users did not use the highlighted terms or link box at all, the majority did use these some of the features and visited a number of external sites via COHSE. 


\section{B. CORESE}

1) Time taken for users to find information/perform tasks: The following table describes the average times for each task and the range of times in minutes for the eleven respondents.

TABLE II. AVERAGE TIMES AND RANGES FOR EACH TASK IN SECONDS

\begin{tabular}{|c|l|l|l|l|}
\hline & \multicolumn{2}{|c|}{ CORESE } & \multicolumn{2}{c|}{ NeLI } \\
\hline & \multicolumn{1}{|c|}{ Task 1 } & \multicolumn{1}{c|}{ Task 2 } & Task 1 & Task 2 \\
\hline Average & 302 & 229 & 317 & 282 \\
\hline Maximum & 471 & 498 & 699 & 472 \\
\hline Minimum & 98 & 78 & 73 & 151 \\
\hline
\end{tabular}

The quickest task on average was Task 2 for the CORESE-based SWB and the longest average task time was Task 1 for NeLI. Six users spent longer on the CORESEbased SWB tasks than on the NeLI tasks and five users spent longer on the NeLI tasks than on the CORESE-based SWB tasks.

2) Use of semantic links compared with non-semantic links: It was not possible to directly compare the use of semantic links with non-semantic links due to the fact that all of the links that a user interacts with on the CORESE- based SWB can be classed as semantic. There were however threehundred and twenty-five searches via the CORESE- based SWB compared to ninety-one searches via NeLI, perhaps indicating that users interacted with the CORESE- based SWB plugin more than they would a standard website.

Once a user has found a document on NeLI via the CORESE-based SWB, the subsequent web pages contain links to external services such as Google and Wikipedia with which users can find more related information. The logs indicate however that respondents did not utilise this feature as only one occurrence was found where the user looked at related information on Wikipedia.

3) Summary: From the log evaluation it is clear that there was little difference in the average time that users spent using the CORESE-based SWB compared to the NeLI website. The second CORESE-based SWB task users did was the fastest overall.

When analyzing the logs, there also seems to have been an issue for users in finding the actual target documents that contained the answers to the tasks. It is possible however that users found documents that contained partial answers that they felt were adequate to answer the task.

\section{GoPubMed}

1) Time taken for users to find information/perform tasks: The average time taken and range of times is shown below for the fifty-two respondents.
TABLE III. AVERAGE TIMES AND RANGES FOR EACH TASK IN SECONDS

\begin{tabular}{|c|l|l|l|l|}
\hline & \multicolumn{2}{|c|}{ GoPubMed } & \multicolumn{2}{c|}{ PubMed } \\
\hline & Task 1 & Task 2 & Task 1 & Task 2 \\
\hline Average & 284 & 174 & 190 & 198 \\
\hline Maximum & 1363 & 796 & 812 & 755 \\
\hline Minimum & 49 & 35 & 12 & 34 \\
\hline
\end{tabular}

The quickest task on average was Task 2 for GoPubMed, which also had the longest average task time for Task 1.

For all tasks the majority of users completed the tasks in less than five minutes but there were some who took considerably longer.

Twenty-nine users spent longer on the GoPubMed tasks than on the PubMed tasks and twenty-three users spent longer on the PubMed tasks compared to the GoPubMed tasks.

2) Use of semantic links compared with non-semantic links: The GoPubMed logs were analysed to determine the levels of use of the semantic links on GoPubMed by identifying clicks on semantic features such as the Tree on the left hand side of the screen which displays semantic information about Authors, Dates and Locations.

From a total of five hundred and ninety-six recorded actions, eighty-six were classed as semantic actions (14\%), generated by twenty-four individual users.

3) Summary: Overall, the quickest average task time was the second tasks that users did using GoPubMed. With the longest task being the first with GoPubMed, this may indicate that there is an initial learning curve for users but once they have understood the system they are better able to utilise its capabilities. The second task however that users did with GoPubMed was around twenty seconds faster on average than the PubMed tasks. Out of the fifty-two logged

users, thirty-six were faster on the second task than the first.

Around forty-six percent of users used the semantic

features at least once indicating that users would use semantic links but as an overall percentage of activity, semantic activity was relatively low.

\section{DISCUSSION}

The following table shows the average times spent using each system and the PubMed and NeLI websites.

TABLE IV. AVERAGE TIME FOR ALL TASKS ON EACH SYSTEM IN SECONDS

\begin{tabular}{|c|c|c|c|c|}
\hline GoPubMed & COHSE & CORESE & PubMed & NeLI \\
\hline 229 & 478 & 266 & 194 & 387 \\
\hline
\end{tabular}

From this, it suggests that tasks using PubMed were the quickest in just over three minutes. The slowest tasks were those done using COHSE in just under eight minutes. The original hypotheses therefore that SWBs reduce the time taken for users to find information then was found to be correct for at least one of the browsers, CORESE, when 
compared with the control system. The fact that the second task performed with CORESE was faster than the first, may indicate that users, once accustomed to navigating using the semantic graph found it easier and were therefore quicker. This however needs further investigation due to the fact that it is not clear whether users found the relevant target documents when performing the tasks with both the SWB and the control system.

Although GoPubMed had a slower average task time, it was shown that for the second task, users performed faster than the control system. Similarly to CORESE, a potential explanation could be that the users who took longer on the first GoPubMed task (which increased the average time) may have just been familiarising themselves with the interface and performed better on the second task.

An important obstacle for both the COHSE and CORESE-based SWBs, (and perhaps a small factor for GoPubMed) identified from user feedback and the logs, was the minimal instructions that were provided in the use of the SWBs. Future work will include carrying out additional workshops to investigate whether a more detailed introduction has an impact on user experience.

With regards to the use of semantic links compared with non-semantic links, when presented with them, the majority of users did use them at least once during the tasks. The range of use was large for all browsers with some users trying the semantic links once whereas some utilised the links frequently. Future work will investigate this difference and to see whether the frequency and type of semantic activity changes the user experience. The ratio of semantic to nonsemantic activity was low for all SWBs which can perhaps be explained by the lack of prior experience with the interfaces combined with the fact that it might not be immediately apparent what the advantage of the use of these "extra" links is to the user.

\section{CONCLUSION}

This paper presented a unique user-centered evaluation of three SWBs investigating the use of semantically provided dynamic links based on underlying domain ontology.

In conclusion, the user experience during the evaluation was dependent on the SWB used but there was some indication that given more exposure to the systems and their semantic features, users will be able to find information more quickly than with the existing non-semantic systems. The use of semantic links was dependent on the individual but with the majority of users utilising them, even if briefly, indicates that users will at least explore these features.

\section{REFERENCES}

[1] Jupp S, Stevens R, Bechhofer S, Yesilada Y \& Kostkova P., "Knowledge Representation for Web Navigation", Semantic Web Applications and Tools for the Life Sciences, 2008.

[2] Hendler J., "Science and The Semantic Web", Science, 2003, 299: p. 24.

[3] Carr L, Bechhofer S, Goble C \& Hall W., "Conceptual Linking: Ontology-based Open Hypermedia", In Proceedings of the 10th international conference on world wide web, 2001.

[4] Bodenreider O \& Stevens R., "Bio-ontologies: current trends and future directions", Briefings in Bioinformatics, 2006, 7: pp. 256-274.

[5] http://www.biotec.tu-dresden.de/sealife.

[6] http://cohse.cs.manchester.ac.uk.

[7] Mrabet Y, Khelif K \& Dieng-Kuntz R., "Recognising professionalactivity groups and web usage mining for web browsing personalisation", In Proceedings of the IEEE/WIC/ACM International Conference on Web Intelligence, 2007.

[8] http://www-sop.inria.fr/edelweiss/software/corese/.

[9] http://www.gopubmed.org.

[10] http://www.ncbi.nlm.nih.gov/pubmed/. 